\title{
A Experiência duma Norma de Atuação no Traumatismo Crânio-Encefálico Ligeiro em Idade Pediátrica: Estudo Longitudinal de Três Anos
}

\author{
The Experience of a Protocol for the Management of \\ Pediatric Minor Head Injury: A Three Years Longitudinal \\ Study
}

\author{
Joana MATIAS $\square^{1}$, Sofia ALMEIDA², Sofia FERRITO ${ }^{1}$, Ana Margarida QUEIROZ ${ }^{1}$, Ana DIAS ALVES ${ }^{3}$, Ana TAVARES $^{4}$, \\ Andreia AMORIM ${ }^{5}$, Paulo CALHAU ${ }^{1}$, Isabel SARAIVA DE MELO 1 \\ Acta Med Port 2017 Oct;30(10):704-712 - https://doi.org/10.20344/amp.8795
}

\section{RESUMO}

Introdução: Os traumatismos crânio-encefálicos são frequentes em Pediatria, habitualmente ligeiros e sem lesões intracranianas. A tomografia computorizada crânio-encefálica é o exame de eleição, implicando radiação ionizante, não existindo consenso nas indicações nos traumatismos crânio-encefálicos ligeiros. Uma atitude expectante é uma opção adequada na maioria dos casos. Os autores pretenderam comparar os doentes internados e submetidos a tomografia computorizada crânio-encefálica com os doentes internados sem tomografia computorizada crânio-encefálica, inferindo a segurança da norma de atuação em vigor na nossa instituição.

Material e Métodos: Estudo analítico longitudinal retrospetivo, durante três anos, de doentes com menos de 15 anos admitidos na Urgência Pediátrica e internados por traumatismos crânio-encefálicos ligeiros. Amostra organizada num grupo A (internados com tomografia computorizada crânio-encefálica) e num grupo B (internados sem tomografia computorizada crânio-encefálica).

Resultados: Amostra de estudo constituída por 206 doentes: 81 (39\%) grupo Ae 125 (61\%) grupo B. Sintomas, nomeadamente vómitos, foram mais frequentes no grupo B $(91 \%$ e $61 \%$ vs $75 \%$ e $35 \%, p<0,05)$; hematoma epicraniano volumoso e afundamento ósseo no grupo $\mathrm{A}(11 \%$ e $12 \%$ vs $0 \%, p<0,05)$. Realizou-se tomografia computorizada crânio-encefálica em $39 \%$ dos doentes estudados (crianças com sinais de alarme à observação ou evolução não favorável durante o internamento); $43 \%$ tinham alterações (29 doentes apresentavam fratura, 18 doentes apresentavam lesões intracranianas). Três doentes foram submetidos a neurocirurgia. Não registámos óbitos, reinternamentos ou sequelas neurológicas.

Discussão: As lesões intracranianas clinicamente significativas foram pouco frequentes. A atitude preconizada de vigilância hospitalar das crianças e adolescentes com traumatismos crânio-encefálicos ligeiros sintomáticos, sem alterações significativas ao exame objetivo, não pareceu ter implicado riscos acrescidos.

Conclusão: A utilização criteriosa da tomografia computorizada crânio-encefálica permitiu reduzir o número de exames e a exposição a radiação ionizante.

Palavras-chave: Criança; Lesões Encefálicas/diagnóstico por imagem; Tomografia Computorizada; Traumatismos Craniocerebrais

\section{ABSTRACT}

Introduction: Head injury is common in children, with mostly being minor and not resulting in intracranial injury. Computerized tomography head scan is the preferred exam, but implies exposure to radiation; the indications for computerized tomography head scan in minor injuries are not consensual. An expectant approach is a good option in most cases. The aim was to compare the patients hospitalized and subjected to computerized tomography head scan with patients hospitalized but not subjected to computerized tomography head scan in order to assess the safety of our institution's practice protocol.

Material and Methods: Analytical longitudinal retrospective study, during three years, including patients younger than 15 years of age with minor head injury, admitted for in hospital surveillance through a paediatric emergency room. We defined two study groups: group A (hospitalized with computerized tomography head scan) and group B (hospitalized without computerized tomography head scan). Results: Study sample consisting of 206 patients: 81 (39\%) group A and $125(61 \%)$ group B. Symptoms, including vomiting, were more frequent in group B $(91 \%$ and $61 \%$ vs $75 \%$ and $35 \%, p<0.05)$; large scalp hematoma and palpable fracture in group A (11\% and $12 \%$ vs $0 \%, p<0.05$ ). We performed computerized tomography head scan in $39 \%$ of the study patients (children with red flags in the physical examination or unfavourable course during hospitalization); $43 \%$ had traumatic brain injury (29 patients had fracture, 18 patients had intracranial injury). Three patients underwent neurosurgery. We did not register deaths, readmissions or neurologic sequelae.

Discussion: Significant intracranial injury was infrequent. The hospitalization and surveillance of children and adolescents with symptomatic minor head injury, without red flags in the physical examination, did not seem to result in additional risks.

Conclusion: The careful selection of patients for computerized tomography head scan enabled a decrease in the number of these exams and the exposure to ionizing radiation.

Keywords: Brain Injuries/diagnostic imaging; Child; Craniocerebral Trauma; Tomography, X-Ray Computed

1. Serviço de Pediatria. Hospital Garcia de Orta. Almada. Portugal.

2. Departamento de Pediatria. Hospital de Santa Maria. Centro Hospitalar de Lisboa Norte. Lisboa. Portugal.

3. Serviço de Pediatria. Hospital dos Lusíadas. Lisboa. Portugal.

4. Serviço de Pediatria. Hospital de Cascais Dr. José de Almeida. Cascais. Portugal.

5. Serviço de Neurocirurgia. Hospital Garcia de Orta. Almada. Portugal.

$\bowtie$ Autor correspondente: Joana Matias. joanagmatias@gmail.com

Recebido: 05 de fevereiro de 2017 - Aceite: 24 de julho de 2017 | Copyright @ Ordem dos Médicos 2017 


\section{INTRODUÇÃO}

Os traumatismos crânio-encefálicos (TCE) são frequentes e potencialmente graves na idade pediátrica, constituindo um problema de saúde pública. ${ }^{1-3} \mathrm{~A}$ incidência real é difícil de apurar. ${ }^{4-6}$ Dados recentes publicados pelo National Institute for Health and Care Excellence apontam para 1400000 admissões na urgência e 200000 internamentos por TCE por ano no Reino Unido, 33\% a $50 \%$ em idades inferiores a 15 anos. ${ }^{7,8} \mathrm{~A}$ incidência atual dos TCE em Portugal não é conhecida, tendo sido de 137 por 100000 habitantes entre 1996 e 1997, para todas as idades..$^{5,6}$

Setenta e cinco a $90 \%$ dos TCE observados na urgência hospitalar em pediatria são ligeiros (isto é, com escala de coma de Glasgow superior ou igual a 13). , $, 4,7,9,10$ As taxas de morbilidade e mortalidade reportadas são baixas; os casos de morte e sequelas ocorrem nos TCE moderados ou graves, com lesão intracraniana (LIC) clinicamente significativa associada. ${ }^{4,7,11}$

A tomografia computorizada crânio-encefálica (TC-CE) é o principal exame na identificação de LIC. ${ }^{11-17} \mathrm{O}$ desafio atual é a identificação dos casos de TCE ligeiro que justificam realizar este exame. ${ }^{3,8,16,18} \mathrm{~A}$ incidência de LIC no TCE ligeiro é muito baixa, sobretudo considerando lesões com necessidade de intervenção cirúrgica urgente. ,., $^{38-21}$ Por outro lado, a radiação ionizante em idade pediátrica tem efeitos deletérios e cumulativos, com risco aumentado de neoplasias (leucemia e tumores cerebrais), atraso global do desenvolvimento, distúrbios endocrinológicos e doença de Moyamoya. Assim, o pedido de exames neuro-imagiológicos nesta população deve ser criterioso. . $^{16,22-25}$

Nos últimos anos, estudos populacionais como PECARN (Pediatric Emergency Care Applied Research Network, elaborado nos Estados Unidos da América), CHALICE (Children's Head Injury Algorithm for the Prediction of Important Clinical Events, elaborado no Reino Unido) e CATCH (Canadian Assessment of Tomography for Childhood Head Injury, elaborado no Canadá) propuseram algoritmos clínicos para o pedido de TC-CE nos TCE ligeiros. ${ }^{8,11,16,19,24-28}$ $A$ atitude preconizada não é uniforme. ${ }^{13,18,20,21,28} \mathrm{O}$ estudo PECARN sugere a realização de TC-CE nas crianças com idade inferior a dois anos e TCE ligeiro associado a escala de coma de Glasgow inferior ou igual a 14, fratura craniana palpável, hematoma occipital, parietal ou temporal, perda de conhecimento superior ou igual a cinco segundos, mecanismo de lesão severo ou suspeita de maus tratos e nas crianças com idade superior ou igual a dois anos e TCE ligeiro associado a escala de coma de Glasgow inferior ou igual a 14 , sinais de fratura da base do crânio, sintomas severos à admissão (perda de conhecimento, vómitos ou cefaleias) associados a agravamento clínico durante o período de observação, preferência do médico ou preferência dos pais. ${ }^{11} \mathrm{O}$ estudo CHALICE preconiza a realização de TC-CE nos TCE ligeiros com perda de conhecimento superior a cinco minutos, amnésia superior a cinco minutos, sonolência excessiva, três ou mais vómitos, suspeita de traumatismo não acidental, convulsão, escala de coma de Glasgow inferior a 14 (ou inferior a 15 em crianças com menos de um ano de idade), suspeita de fratura craniana deprimida, fontanela abaulada, sinais de fratura da base do crânio, sinais neurológicos focais, hematoma ou escoriação do couro cabeludo com dimensão superior a $5 \mathrm{~cm}$ nas crianças com menos de um ano de idade ou mecanismo de lesão de elevado impacto (acidente de viação a alta velocidade como pedestre, ciclista ou ocupante de veículo, queda de altura superior a três metros e traumatismo por objeto projetado a alta velocidade). ${ }^{25} \mathrm{O}$ estudo $\mathrm{CATCH}$ recomenda a realização de TC-CE nos TCE ligeiros com escala de coma de Glasgow inferior a 15 duas horas após o TCE, suspeita de fratura craniana aberta ou deprimida, história de cefaleias de agravamento progressivo, irritabilidade ao exame objetivo, sinais de fratura da base do crânio, hematoma volumoso do couro cabeludo ou mecanismo de lesão de elevado impacto (acidente de viação, queda de altura superior ou igual a $91 \mathrm{~cm}$ ou queda de bicicleta sem capacete)..$^{19}$

Estudos recentes demonstram que a presença de sonolência, vómitos ou cefaleias não se associa a uma maior probabilidade de LIC clinicamente significativa, sobretudo quando estes sintomas são transitórios e autolimitados. ${ }^{3,13,17,30,33,34}$

Em Portugal, a literatura neste âmbito é ainda escassa.

Desde 2004 vigora na nossa instituição uma norma de atuação nos TCE ligeiros que prevê o internamento e vigilância em meio hospitalar dos TCE ligeiros com determinados sinais de alarme na avaliação inicial, em alternativa à TC-CE imediata (Tabela 1).

Os autores propõem-se estudar a amostra de crianças e adolescentes internados para vigilância por TCE ligeiro numa urgência pediátrica polivalente. Como objetivo principal, pretendeu-se comparar as características clínicas e a evolução do grupo de doentes internados e submetidos a TC-CE com o grupo de doentes internados e não submetidos a TC-CE, inferindo-se a segurança da norma de atuação em vigor. Como objetivos secundários, pretendeu-se avaliar a frequência de fratura craniana e/ou LIC e avaliar a utilidade da radiografia de crânio nos TCE ligeiros internados.

\section{MATERIAL E MÉTODOS}

Realizámos um estudo analítico longitudinal retrospetivo durante três anos (1/1/2011 a 31/12/2013) de uma amostra de doentes com idade inferior a 15 anos admitidos por TCE ligeiro na Urgência Pediátrica de um hospital de nível Il com urgência polivalente (incluindo Neurocirurgia) e internados para vigilância na Unidade de Internamento de Curta Duração (UICD) da Urgência Pediátrica.

Definimos TCE ligeiro como traumatismo craniano fechado com nível de consciência normal ou ligeiramente alterado (13 a 15 na escala de coma de Glasgow) na primeira avaliação hospitalar. Definimos LIC clinicamente significativa, à semelhança dos autores do PECARN, como lesão intracraniana que conduz ao óbito, que implica intervenção cirúrgica, que se associa a ventilação invasiva por 
Tabela 1 - Norma de atuação clínica da Urgência Pediátrica da nossa instituição hospitalar no traumatismo crânio-encefálico ligeiro (isto é, com escala de coma de Glasgow 13 a 15 na avaliação inicial)

Alta para o domicílio sem exames complementares (vigilância de sinais de alarme):

- Mecanismo de baixa intensidade

- Escala de coma de Glasgow 15

- Estabilidade hemodinâmica

- Exame neurológico sem alterações

- Sem sinais indiretos de fratura do crânio (hematoma volumoso e idade inferior a dois anos, afundamento ósseo, hematoma periorbitário bilateral, hematoma retroauricular, rinorráquia, otorráquia, otorragia e hemotímpano)

Internamento na UICD (analgesia, monitorização e vigilância clínica):

- Escala de coma de Glasgow 13 ou 14

- Perda de conhecimento inferior a 5 minutos

- Amnésia

- Vómitos persistentes

- Cefaleias persistente

TC-CE:

- Perda de conhecimento superior a 5 minutos

- Convulsão após o traumatismo craniano

- Suspeita de maus tratos

- Sinais neurológicos focais

- Sinais indiretos de fratura do crânio

- Radiografia do crânio sugestivo de fratura

- Agravamento clínico durante o internamento na UICD

\section{Radiografia do crânio:}

- Idade inferior a dois anos e hematoma epicraniano volumoso

UICD: unidade de internamento de curta duração; TC-CE: tomografia computorizada crânio-encefálica

um período superior a 24 horas ou que se associa a um internamento hospitalar prolongado (igual ou superior a duas noites) para vigilância clínica ou imagiológica. ${ }^{11}$

A norma de orientação clínica nos TCE da Urgência Pediátrica, elaborada por um grupo de Pediatras experientes do serviço (com base na evidência científica disponível) em colaboração com a equipa de Neurocirurgia da nossa instituição, aprovada por todo o serviço de Pediatria e implementada em 2004 (Tabela 1), preconiza:

- Internamento em UICD com vigilância clínica (monitorização da frequência cardíaca, tensão arterial e escala de coma de Glasgow) e analgesia nos TCE ligeiros com escala de coma de Glasgow de 13 ou 14, perda de conhecimento inferior a cinco minutos, amnésia, vómitos persistentes ou cefaleias persistentes.

- Realização de TC-CE nos TCE ligeiros com perda de conhecimento superior a cinco minutos, sinais neurológicos focais, convulsão após TCE, sinais indiretos de fratura craniana, radiografia de crânio com sinais de fratura ou agravamento clínico durante a vigilância hospitalar. São considerados sinais clínicos indiretos de fratura craniana hematoma volumoso do couro cabeludo (sobretudo em crianças com idade inferior a dois anos), hematoma periorbitário bilateral ou retroauricular, presença de rinorráquia, otorráquia, otorragia ou hemotímpano e noção de afundamento ósseo.

- Realização de radiografia de crânio no TCE ligeiro em crianças com idade inferior a dois anos e hematoma epicraniano volumoso.

- Alta para o domicílio sem realização de exames complementares de diagnóstico e com indicação para vigilância dos sinais de alarme nos casos de TCE com mecanismo de baixa intensidade, escala de coma de Glasgow na admissão de 15, estabilidade hemodinâmica, exame neurológico sem alterações e ausência de sinais indiretos de fratura do crânio.

Considerámos como critérios de inclusão: doentes internados na UICD da Urgência Pediátrica da nossa instituição durante o período de estudo com o diagnóstico de TCE. Definimos como critérios de exclusão: escala de coma de Glasgow inferior ou igual a 12 na primeira avaliação hospitalar, transferência de outro hospital para avaliação por Neurocirurgia, antecedentes de doença neurológica crónica, discrasia hemorrágica e uso de fármacos anticoagulantes ou antiagregantes plaquetários.

Os doentes foram identificados a partir da base de dados da UICD (registo informático com número de processo, identificação e diagnóstico dos doentes internados). RecoInemos os dados consultando os processos clínicos hospitalares informatizados (o episódio de urgência do TCE e um episódio de urgência e/ou consulta hospitalar subsequente para excluir reinternamentos e sequelas neurológicas, nos casos com pelo menos uma observação posterior na nossa instituição). A partir dos dados do sistema de informação para a gestão de doentes da nossa instituição registámos as TC-CE realizadas sem internamento em UICD.

A amostra de estudo foi dividida em dois grupos: doentes internados com TC-CE (grupo A) e doentes internados sem TC-CE (grupo B).

Analisámos variáveis demográficas (sexo e idade) e clínicas: mecanismo de lesão (queda da própria altura, queda de altura inferior ou superior a três metros, atropelamento, acidente de viação como ciclista, acidente de viação como passageiro ou outro mecanismo de lesão); localização do 
traumatismo (frontal, parietal, temporal, occipital ou múltipla); sintomas após o TCE (vómitos, sonolência, cefaleias, perda de conhecimento inferior ou superior a cinco minutos, amnésia, tonturas, alterações da visão ou desorientação); alterações no exame objetivo inicial (hematoma epicraniano inferior ou superior a cinco centímetros, afundamento ósseo, escoriações ou sinais neurológicos focais).

Registámos ainda dados imagiológicos: radiografias de crânio realizadas e alterações encontradas (presença ou não de sinais de fratura), TC-CE realizadas e alterações encontradas (fratura craniana, hematoma epidural, hematoma subdural ou contusão cerebral). Documentámos o local do internamento (UICD, Enfermaria de Pediatria e/ou Unidade de Cuidados Intensivos Pediátricos), a evolução clínica (persistência ou agravamento dos sintomas iniciais, deterioração do estado de consciência ou aparecimento de sinais neurológicos de novo), realização de intervenção neurocirúrgica, reinternamento e presença de sequelas pós TCE (nos casos com observação posterior, na Urgência Pediátrica ou em consulta, no nosso hospital).

Realizou-se uma análise estatística univariada utilizando-se o teste qui-quadrado para comparação de variáveis categóricas de dois grupos independentes e o teste de Mann-Whitney para comparação de variáveis numéricas de dois grupos independentes. Considerou-se um limiar de significância de 0,05 . Utilizou-se o programa informático IBM $^{\circledR}$ SPSS $^{\circledR}$ Statistics 20.0 for Windows ${ }^{\circledR}$.

\section{RESULTADOS}

Nos três anos estudados foram admitidos na Urgência Pediátrica do nosso hospital 128554 crianças e adolescentes com idade inferior a 15 anos. Cerca de 3\% do total das admissões (3393 / 128 554) apresentavam TCE (diagnóstico de traumatismo craniano não especificado: a designação genérica utilizada pelos Pediatras do serviço responsáveis pela codificação dos diagnósticos dos doentes admitidos na Urgência Pediátrica). Oito por cento dos TCE (285 / 3393) foram internados na UICD (Fig. 1). Excluímos da nossa amostra inicial 79 indivíduos (28\%): 55 transferidos de outro hospital para avaliação por Neurocirurgia, 13 com valor inferior a 13 na Escala de Coma de Glasgow na admissão hospitalar (ou seja, TCE não ligeiro), nove com antecedentes de doença neurológica crónica e dois com antecedentes de discrasia hemorrágica.

Noventa e oito porcento das crianças e adolescentes avaliados neste período na Urgência Pediátrica por traumatismo craniano apresentavam TCE ligeiro (3314 / 3393). Assim, a taxa de internamento nos doentes com TCE ligeiro foi de $6 \%$ (206 / 3314). Além das crianças internadas na UICD, 40 doentes realizaram TC-CE na Urgência Pediátrica sem internamento (1,2\% do total de TCE ligeiros), respeitando-se o protocolo em vigor no nosso serviço. No total, 3,6\% (121 / 3314) dos doentes com TCE ligeiro avaliados na Urgência Pediátrica durante o período do estudo realizaram TC-CE.

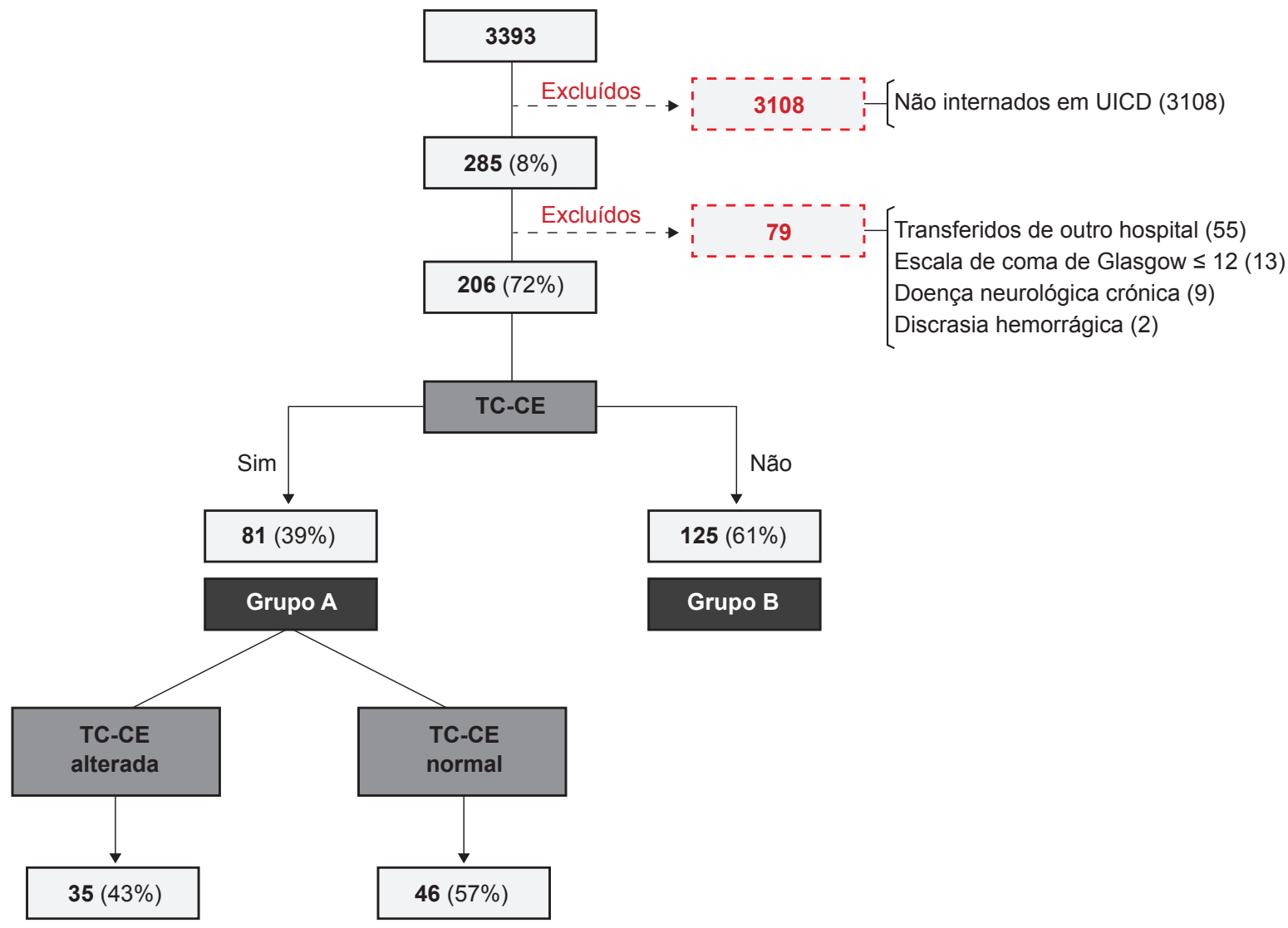

Figura 1 - Constituição da amostra total e dos grupos de estudo. No período estudado foram admitidos na Urgência Pediátrica 3393 doentes com o diagnóstico de traumatismo craniano não especificado.

UICD: unidade de internamento de curta duração; TC-CE: tomografia computorizada crânio-encefálica 
A amostra final ficou assim constituída por 206 doentes, posteriormente classificados em dois grupos (Fig. 1): doentes internados e submetidos a TC-CE (grupo $A, n=81$ ) e doentes internados não submetidos a TC-CE (grupo $B, n=$ 125). Os dados demográficos, clínicos e imagiológicos da amostra e grupos de estudo são apresentados na Tabela 2.

Considerando a amostra total, 61\% (126 / 206) das crianças e adolescentes estudados eram do sexo masculino. A mediana da idade foi de 6,5 anos (mínimo: 11 dias, máximo: 14 anos e 11 meses), média 6,32 anos (desvio padrão: 4,78 anos). Trinta e um por cento dos doentes (64 / 206) tinha menos de dois anos de idade. Não encontrámos diferenças significativas quanto ao sexo e distribuição etária entre os dois grupos de estudo.

Os mecanismos de lesão mais frequentes foram a que- da de altura inferior a três metros (34\%) e a queda da própria altura (28\%). Os acidentes de viação (atropelamento, acidente como ciclista ou como ocupante de veículo) corresponderam a $18 \%$. Entre os outros mecanismos verificou-se um caso de uma criança de 18 meses agredida na cabeça, face e tronco com golpes de mão fechada (vítima de maus tratos). A queda da própria altura foi significativamente mais frequente nos doentes não submetidos a TC-CE: 38\% (48 / 125) no grupo B versus 11\% (9 / 81) no grupo $\mathrm{A}(p<0,001)$. A queda de altura superior a três metros foi significativamente mais frequente no grupo submetido a TC-CE (grupo A): $6 \%(5 / 81)$ vs $1 \%(1 / 81)$ no grupo B ( $p$ $=0,023)$; também 'outro mecanismo' foi significativamente mais frequente no grupo que realizou TC-CE, 26\% (21 / 81) no grupo A vs 10\% (13/125) no grupo B $(p=0,009)$.

Tabela 2 - Caracterização da amostra total (doentes com traumatismo crânio-encefálico ligeiro internados para vigilância na Unidade de Internamento de Curta Duração) e dos grupos de estudo: grupo A (doentes internados e submetidos a tomografia computorizada crânio-encefálica) e grupo B (doentes internados, mas não submetidos a tomografia computorizada crânio-encefálica)

\begin{tabular}{|c|c|c|c|c|}
\hline & Total $(n=206)$ & Grupo A $(n=81)$ & Grupo B $(n=125)$ & Valor $p$ \\
\hline Idade [Média \pm Desvio padrão (anos)] & $6,32 \pm 4,78$ & $6,00 \pm 5,20$ & $6,52 \pm 4,48$ & NS \\
\hline Sexo masculino [n (\%)] & $126(61 \%)$ & $51(63 \%)$ & $75(60 \%)$ & NS \\
\hline $\begin{array}{l}\text { Mecanismo de lesão [n (\%)] } \\
\text { - Queda própria altura } \\
\text { - Queda < } 3 \text { metros } \\
\text { - Queda > } 3 \text { metros } \\
\text { - Atropelamento } \\
\text { - Acidente viação ciclista } \\
\text { - Acidente viação passageiro } \\
\text { - Outro mecanismo }\end{array}$ & $\begin{array}{c}57(28 \%) \\
70(34 \%) \\
6(3 \%) \\
17(8 \%) \\
7(3 \%) \\
15(7 \%) \\
34(17 \%)\end{array}$ & $\begin{array}{c}9(11 \%) \\
26(32 \%) \\
5(6 \%) \\
10(12 \%) \\
3(4 \%) \\
7(9 \%) \\
21(26 \%)\end{array}$ & $\begin{array}{c}48(38 \%) \\
44(35 \%) \\
1(1 \%) \\
7(6 \%) \\
4(4 \%) \\
8(6 \%) \\
13(10 \%)\end{array}$ & $\begin{array}{l}<0,001 \\
\text { NS } \\
0,023 \\
\text { NS } \\
\text { NS } \\
\text { NS } \\
0,009\end{array}$ \\
\hline $\begin{array}{l}\text { Localização do TCE [n (\%)] } \\
\text { - Frontal } \\
\text { - Parietal } \\
\text { - Temporal } \\
\text { - Occipital } \\
\text { - Múltipla }\end{array}$ & $\begin{array}{c}108(52 \%) \\
71(34 \%) \\
16(8 \%) \\
62(30 \%) \\
53(26 \%)\end{array}$ & $\begin{array}{l}38(47 \%) \\
43(53 \%) \\
11(14 \%) \\
23(28 \%) \\
30(37 \%)\end{array}$ & $\begin{array}{c}70(56 \%) \\
28(22 \%) \\
5(4 \%) \\
39(31 \%) \\
23(18 \%)\end{array}$ & $\begin{array}{c}\text { NS } \\
<0,001 \\
0,012 \\
\text { NS } \\
0,003\end{array}$ \\
\hline $\begin{array}{l}\text { Assintomático [n (\%)] } \\
\text { Sintomático [n (\%)] } \\
\text { - Vómitos } \\
\text { - Sonolência } \\
\text { - Cefaleias } \\
\text { - Perda conhecimento < } 5 \text { minutos } \\
\text { - Perda conhecimento > } 5 \text { minutos } \\
\text { - Amnésia } \\
\text { - Tonturas } \\
\text { - Alterações da visão } \\
\text { - Desorientação }\end{array}$ & $\begin{array}{c}31(15 \%) \\
175(85 \%) \\
104(50 \%) \\
98(48 \%) \\
51(25 \%) \\
38(18 \%) \\
2(1 \%) \\
35(17 \%) \\
13(6 \%) \\
4(2 \%) \\
7(3 \%)\end{array}$ & $\begin{array}{c}20(25 \%) \\
61(75 \%) \\
28(35 \%) \\
37(46 \%) \\
16(20 \%) \\
17(21 \%) \\
0(0 \%) \\
17(21 \%) \\
5(6 \%) \\
1(1 \%) \\
4(5 \%)\end{array}$ & $\begin{array}{c}11(9 \%) \\
114(91 \%) \\
76(61 \%) \\
61(49 \%) \\
35(28 \%) \\
23(18 \%) \\
2(2 \%) \\
18(14 \%) \\
8(6 \%) \\
3(2 \%) \\
3(2 \%)\end{array}$ & $\begin{array}{c}<0,001 \\
<0,001 \\
0,001 \\
N S \\
N S \\
N S \\
\text { NS } \\
\text { NS } \\
\text { NS } \\
\text { NS } \\
\text { NS }\end{array}$ \\
\hline $\begin{array}{l}\text { Observação inicial normal [n (\%)] } \\
\text { Observação inicial alterada [n (\%)] } \\
\text { - Hematoma }<5 \mathrm{~cm} \\
\text { - Hematoma }>5 \mathrm{~cm} \\
\text { - Afundamento ósseo } \\
\text { - Escoriações } \\
\text { - Sinais neurológicos focais }\end{array}$ & $\begin{array}{c}62(30 \%) \\
144(70 \%) \\
104(50 \%) \\
9(4 \%) \\
10(5 \%) \\
42(20 \%) \\
5(2 \%)\end{array}$ & $\begin{array}{c}18(22 \%) \\
63(78 \%) \\
42(52 \%) \\
9(11 \%) \\
10(12 \%) \\
17(21 \%) \\
4(5 \%)\end{array}$ & $\begin{array}{c}44(35 \%) \\
81(65 \%) \\
62(50 \%) \\
0 \\
0 \\
25(20 \%) \\
1(1 \%)\end{array}$ & $\begin{array}{l}\text { NS } \\
\text { NS } \\
\text { NS } \\
0,001 \\
<0,001 \\
\text { NS } \\
\text { NS }\end{array}$ \\
\hline $\begin{array}{l}\text { Radiografia de crânio [n (\%)] } \\
\text { - Normal } \\
\text { - Sinais de fratura }\end{array}$ & $\begin{array}{c}44(21 \%) \\
29(14 \%) \\
15(7 \%)\end{array}$ & $\begin{array}{c}20(25 \%) \\
5(6 \%) \\
15(19 \%)\end{array}$ & $\begin{array}{c}24(19 \%) \\
24(19 \%) \\
0\end{array}$ & $\begin{aligned} & N S \\
< & 0,001 \\
< & 0,001\end{aligned}$ \\
\hline Intervenção neurocirúrgica [n (\%)] & $3(2 \%)$ & $3(4 \%)$ & 0 & 0,029 \\
\hline Sequelas [n (\%)] & 0 & 0 & 0 & NS \\
\hline
\end{tabular}

TCE: traumatismo crânio-encefálico; NS: diferença estatística não significativa (valor $p>0,05$ ) 
$\mathrm{Na}$ amostra total, a localização mais frequente do TCE foi frontal (52\% dos casos). A localização parietal foi significativamente mais frequente no grupo A (submetido a TC-CE) com 53\% (43/81) vs 22\% (28/125) no grupo B ( $p$ $<0,001)$, tal como a localização temporal com 14\% (11/ 81) no grupo $A$ vs $4 \%(5 / 125)$ no grupo $B(p=0,012)$ e a localização múltipla do TCE com 37\% (30 / 81) no grupo A e $18 \%(23 / 125)$ no grupo B $(p=0,003)$.

Após o TCE, $85 \%$ dos doentes apresentava sintomas: $50 \%$ vómitos (104 / 206), 48\% sonolência (98 / 206) e 25\% cefaleias (51 / 206). A presença de sintomas foi significativamente mais frequente nos doentes internados sem TC-CE: $91 \%$ no grupo B (114 / 125) e 75\% (61 / 81) no grupo $\mathrm{A}(p<0,001)$, nomeadamente a presença de vómitos: $61 \%$ no grupo $\mathrm{B}(76 / 125)$ vs $35 \%(28 / 81)$ no grupo $\mathrm{A}(p=$ 0,001).

Setenta por cento dos doentes apresentava alterações no exame objetivo inicial: hematoma epicraniano em 55\% (113 / 206) foi a alteração mais frequente. A presença de hematoma com mais de cinco centímetros de maior diâmetro foi significativamente mais frequente no grupo que realizou TC-CE: $11 \%$ do grupo A (9/81) vs 0\% (0/125) do grupo $B(p=0,001)$; também a noção de afundamento ósseo foi mais frequente no primeiro grupo: 12\% (10 / 81) no grupo A vs $0 \%(0 / 125)$ no grupo $\mathrm{B}(p<0,001)$. Relativamente ao exame neurológico inicial, apenas cinco doentes apresentavam sinais focais; todos realizaram TC-CE exceto uma criança de oito anos com dismetria na prova dedo-nariz que resolveu durante a primeira hora de internamento em UICD. Houve dois doentes com perda de conhecimento superior a cinco minutos que não realizaram TC-CE; ambos apresentaram uma evolução clínica favorável durante o período de vigilância.

Os doentes submetidos a TC-CE (grupo A) realizaram este exame em média 4,95 horas após o traumatismo craniano. Quarenta e três por cento (35/81) apresentavam alterações neuro-imagiológicas (Tabela 3): fratura craniana em 36\% (29 / 81) e LIC em 25\% (20 / 81). Os casos de LIC corresponderam a hematomas epidurais (9), hematomas subdurais (7) e contusões cerebrais (4). Setenta e sete por cento (27 / 35) das TC-CE alteradas ocorreram no grupo de crianças com idade inferior a dois anos $(p<0,001)$.

Vinte e um por cento das crianças (44 / 206) realizaram radiografia de crânio.

Tabela 3 - Resultados das tomografias computorizadas crânio-encefálicas (TC-CE) realizadas nos doentes do grupo A (doentes com TCE ligeiro internados e submetidos a tomografia computorizada crânio-encefálica) e principais alterações

\begin{tabular}{lc}
\hline TC-CE & $\mathbf{n = 8 1}$ \\
\hline Normal & $46(57 \%)$ \\
Alterada & $35(43 \%)$ \\
- Fratura crânio & $29(36 \%)$ \\
- Hematoma epidural & $9(11 \%)$ \\
- Hematoma subdural & $7(9 \%)$ \\
- Contusão cerebral & $2(5 \%)$ \\
\hline
\end{tabular}

Neste grupo, 22 doentes tinham idade inferior a dois anos e 17 tinham um hematoma epicraniano à observação (um com hematoma superior a cinco centímetros). Isto é, dos doentes que realizaram radiografia de crânio, 53\% (23 / 44) tinham critérios para tal segundo o protocolo em vigor. Em 34\% das crianças com radiografia (15/44) foram detetadas imagens sugestivas de fratura; todas estas realizaram TC-CE que confirmou fratura nos 15 casos. Associado à fratura, seis destes casos tinham hematoma epidural e dois casos hematoma subdural na TC-CE. Por outro lado, a radiografia não identificou dois casos de fratura da base do crânio (ambos associados a LIC), reconhecidas apenas na TC-CE: uma criança de oito anos que realizou TC-CE por agravamento dos vómitos durante o período de vigilância clínica e uma criança de seis anos com hematoma epicraniano à observação que acabou por realizar TC-CE pela exuberância do hematoma; nenhum destes casos apresentava sinais clínicos sugestivos de fratura da base do crânio.

Noventa e dois por cento (190 / 206) das crianças e adolescentes da amostra estudada foi internada exclusivamente na UICD da Urgência Pediátrica, permanecendo em vigilância menos de 24 horas. Dezasseis doentes do grupo A, com TC-CE alterada, necessitaram de vigilância hospitalar por um período superior: um na Unidade de Cuidados Intensivos Pediátricos (criança de seis anos com fratura e hematoma epidural sem indicação cirúrgica) e 15 na enfermaria de Pediatria.

Três doentes (1,5\% da amostra e $0,1 \%$ do total de TCE ligeiros observados no período estudado) foram submetidos a cranioplastia para correção do defeito ósseo (fratura): lactente de cinco meses com TCE parietal (queda de altura inferior a três metros), perda de conhecimento inferior a cinco minutos, hematoma epicraniano inferior a $5 \mathrm{~cm}$ com afundamento ósseo e TC-CE com fratura parietal isolada; lactente de 10 meses com TCE frontoparietal (queda de altura inferior a três metros), vómitos incoercíveis, sem alterações ao exame objetivo e TC-CE com fratura parietal e hematoma epidural; lactente de 10 meses com TCE parietal (embate direto em superfície dura) assintomático, noção de afundamento ósseo à observação e TC-CE com fratura parietal isolada. Os três tiveram uma evolução clínica favorável, sem intercorrências no período pós-operatório.

Não houve óbitos na amostra durante o período do estudo.

Analisámos o episódio de urgência e de consulta hospitalar subsequente ao TCE em mais de $95 \%$ dos doentes. Em nenhum destes casos se verificou reinternamento na nossa instituição hospitalar relacionado com o traumatismo craniano ou evidência de sequelas neurológicas.

\section{DISCUSSÃO}

No período do estudo, o traumatismo craniano foi um motivo frequente de recurso à Urgência Pediátrica, sendo em 98\% dos casos TCE ligeiros (3314 / 3393). Esta proporção foi superior à descrita na literatura que refere que $75 \%$ a $90 \%$ dos TCE observados na urgência hospitalar em pediatria são ligeiros. ${ }^{2,4,7,9,10}$ 
A amostra estudada, constituída pelos doentes internados na UICD por TCE ligeiro, correspondeu a $6 \%$ do total de TCE observados nesse período (206 / 3393). Tal como documentado na literatura, a maioria dos doentes eram do sexo masculino e mais de um quarto da amostra tinha idade inferior a dois anos..$^{1,4,13,14,18,29}$ Apenas 39\% (81/206) destes doentes realizaram TC-CE. ${ }^{11,13,21}$

Comparando as características clínicas dos dois grupos de estudo, o grupo de doentes internados e submetidos a TC-CE apresentou mecanismos de lesão de maior impacto que o grupo sem TC-CE. A localização parietal, temporal ou múltipla dos TCE associou-se de forma significativa à realização de TC-CE. Embora a localização do traumatismo não esteja discriminada na norma de atuação clínica em vigor na nossa instituição, estas localizações associam-se mais frequentemente à presença de hematoma volumoso e/ou sinais de afundamento à observação e, como tal, a maior risco de fratura, justificando a realização de TC-CE. Tal poderá ser explicado pelo mecanismo de lesão habitualmente associado aos TCE com estas localizações: quedas desamparadas para trás e acidentes de viação, por exemplo.

Os sintomas pós-traumatismo foram mais frequentes no grupo não submetido a TC-CE (sobretudo vómitos, mas também cefaleias). Esta diferença justifica-se pelo facto de se privilegiar o internamento e vigilância das crianças sintomáticas à admissão (desde que sem sinais de alarme ao exame objetivo), em detrimento da avaliação neuro-imagiológica precoce. Nestes doentes, verificou-se uma melhoria clínica durante o período de vigilância e após analgesia, abdicando-se por isso da TC-CE. De facto, é habitual surgirem vómitos após um TCE ligeiro mas, na idade pediátrica, vários estudos sugerem que esse sintoma não é um forte indicador para presença de LIC. 3,13,17,30 Queixas de cefaleias são também frequentes, sendo muitas vezes intensas, persistentes e difíceis de tratar. ${ }^{6,10,31,32,33}$ No entanto, vários trabalhos comprovam que este sintoma, na ausência de outros sinais de alarme, também não se associa a lesões traumáticas clinicamente significativas. ${ }^{33,34}$

Por outro lado, a presença de alterações ao exame objetivo, sobretudo hematoma epicraniano volumoso (superior a cinco centímetros) e noção de afundamento ósseo, mostrou estar associada à realização de TC-CE. Tal demonstra o cumprimento da norma de atuação em vigor na Urgência Pediátrica. As crianças não submetidas a TC-CE apresentavam alterações mínimas ao exame objetivo (quase exclusivamente escoriações e pequenos hematomas do couro cabeludo). Vários estudos afirmam que hematomas volumosos e noção de afundamento ósseo são fatores de risco para fratura e/ou LIC. .,13,19,35 $\mathrm{Na}$ verdade, estes sinais são considerados de alarme pelos três algoritmos de decisão clínica mais utilizados internacionalmente na abordagem dos TCE ligeiros em idade pediátrica (PECARN, CHALICE e CATCH). ${ }^{11,19,25}$

Não podemos deixar de comentar que duas crianças com perda de conhecimento superior a cinco minutos e uma criança com sinais focais à admissão não realizaram
TC-CE, não se respeitando o protocolo em vigor na nossa instituição. Ainda assim, a sua evolução foi favorável e aparentemente sem sequelas neurológicas. Por outro lado, apenas metade das crianças que realizaram radiografia de crânio tinham critérios para tal, segundo o nosso protocolo. Este facto parece traduzir práticas antigas, não baseadas na evidência mais atual e não contempladas na norma de atuação instituída.

Quarenta e três por cento das TC-CE realizadas (35 / 81) e 17\% (35/206) da amostra de estudo (TCE ligeiros internados) apresentavam LIC e/ou fratura de crânio. A comparação destes resultados com os apresentados pela literatura é complexa, visto a definição das amostras de estudo ser heterogénea. A maioria dos trabalhos refere-se à frequência de fratura e/ou LIC na amostra de crianças com TCE ligeiro que realizou TC-CE (frequência de aproximadamente 5\%). ${ }^{18,30}$ As grandes séries internacionais, como os estudos PECARN e CATCH, também apresentam frequências inferiores de TC-CE alterada. O estudo PECARN refere a presença de alterações na TC-CE (hemorragia ou contusão intracraniana, edema cerebral, enfarte traumático, lesão axonal difusa, trombose do seio venoso, desvio da linha média ou sinais de herniação cerebral, diástase das suturas, pneumocéfalo e fratura craniana deprimida) em quatro a $10 \%$ dos TCE ligeiros. O estudo $\mathrm{CATCH}$ refere presença de alterações na TC-CE (hematoma epidural, contusão cerebral, pneumocéfalo, hematoma subdural, fratura craniana deprimida, hemorragia subaracnoideia, hematoma intracerebral, edema cerebral difuso, hematoma cerebeloso e hemorragia intraventricular) em quatro por cento dos TCE ligeiros. ${ }^{11,19}$ A maior frequência de TC-CE alterada do nosso trabalho é indicadora do rigoroso critério de seleção dos doentes que realizaram exame neurorradiológico. Os nossos resultados são tanto mais relevantes quanto não se verificou nenhum reinternamento ou sequela neurológica, pelo menos nos doentes em que foi possível analisar um episódio de urgência ou consulta subsequente no nosso hospital (mais de $95 \%$ da amostra total).

$\mathrm{Na}$ TC-CE a alteração mais frequentemente documentada foi fratura craniana em $36 \%(29 / 81)$ do grupo submetido a TC-CE e 14\% (29 / 206) da amostra de estudo. A frequência de LIC foi de $22 \%$ (18 / 81) do grupo submetido a TC-CE e $9 \%$ (18 / 206) da amostra de estudo. Tal como relatado por outros trabalhos, a maioria dos doentes com TC-CE alterada tinha idade inferior a dois anos..$^{9,14}$ Neste grupo etário, a avaliação clínica é mais difícil, há maior risco de fratura craniana e LIC clinicamente significativa (mesmo se TCE ligeiro) e a LIC é muitas vezes assintomática. Por outro lado, o risco de maus tratos é maior. ${ }^{1,3,16}$

A radiografia craniana foi realizada num número significativo de crianças e levou à suspeita de fratura em 15 doentes; oito destes apresentavam LIC associada na TC-CE. Dois doentes com fratura da base do crânio e LIC na TC-CE tinham radiografia normal. Efetivamente a interpretação dos achados da radiografia de crânio nem sempre é fácil e este exame associa-se a poucos benefícios na avaliação inicial dos TCE ligeiros, uma vez que a presença de 
imagens sugestivas de fratura não permite presumir a presença de LIC e uma radiografia normal não permite excluí-la. 3,9,14 A utilidade da radiografia de crânio na avaliação das crianças e adolescentes com TCE parece ser, à luz destes dados, reduzida. Assim, propomos que crianças com idade inferior a dois anos e hematoma craniano volumoso associado deverão realizar TC-CE. A radiografia de crânio poderá ter alguma aplicação em instituições sem possibilidade de realizar TC-CE. 3,6,14

Quanto ao tratamento e evolução no internamento, apenas $1,5 \%$ dos doentes da amostra (3 / 206) e 0,1\% (3 / 3314) do total de TCE ligeiros avaliados na Urgência Pediátrica nos três anos estudados, foram submetidos a intervenção neurocirúrgica. Este resultado é inferior aos publicados na literatura ( $1 \%$ a $5 \%)$. $^{3,11,13,30}$ Tal ilustra o baixo risco de lesões cranianas graves nos TCE ligeiros em idade pediátrica.

A duração recomendada da vigilância hospitalar ainda não é consensual. Neste estudo foi ponderada caso a caso, em função da evolução clínica. Alguns trabalhos comprovam que um período de seis horas, intervalo em que as LIC hipertensivas se podem manifestar, será suficiente. ${ }^{15,16,}$

Por fim, importa referir as limitações metodológicas do presente estudo. É um estudo retrospetivo, baseado em dados recolhidos de processos clínicos informáticos por vezes incompletos. A amostra estudada é pequena e o grupo de crianças com TCE ligeiro não internadas, o maior grupo de doentes com esse diagnóstico, não foi analisado. Por último, a estratégia utilizada de procurar um episódio de urgência ou consulta subsequente para exclusão de sequelas neurológicas, apesar da maioria dos doentes ter pelo menos uma observação posterior no nosso serviço, não permite a identificação de sequelas ligeiras, sequelas de manifestação tardia e sequelas em doentes que não voltaram a ser observados na nossa unidade hospitalar.

É fundamental a realização de novos estudos, idealmente com uma amostra de maior dimensão, prospetivos e que incluam uma avaliação neurológica ou neuropsicológica posterior para excluir a presença de sequelas, que permitam verificar a segurança desta atitude expectante e determinar a duração ótima do período de vigilância hospitalar.

\section{REFERÊNCIAS}

1. Atabaki SM. Pediatric head injury. Pediatr Rev. 2007;28:215-24.

2. Cassidy JD, Carroll LJ, Peloso PM, Borg J, Holst H, Holm L, et al. Incidence, risk factors and prevention of mild traumatic brain injury: Results of the WHO collaborating centre task force on mild traumatic brain injury. J Rehabil Med Suppl. 2004;43:28-60.

3. Schutzman SA, Greenes DS. Pediatric minor head trauma. Ann Emerg Med. 2001;37:65-74.

4. Trefan L, Houston R, Pearson G, Edwards R, Hyde P, Maconochie I, et al. Epidemiology of children with head injury: a national overview. Arch Dis Child. 2015;0:1.6.

5. Santos ME, Sousa L, Castro-Caldas A. Epidemiologia dos traumatismos crânio-encefálicos em Portugal. Acta Med Port. 2003;16:71-6.

6. Oliveira E, Lavrador JP, Santos MM, Lobo Antunes J. Traumatismo crânio-encefálico: abordagem integrada. Acta Med Port. 2012;25:17992.

7. Head injury: triage, assessment, investigation and early management of

\section{CONCLUSÃO}

A ocorrência de LIC nos TCE ligeiros, sobretudo de LIC clinicamente significativa, é pouco frequente. A utilização criteriosa da TC-CE nos doentes com TCE ligeiro e alterações relevantes ao exame objetivo ou não resolução dos sintomas em internamento, permitiu uma maior percentagem de diagnóstico de lesão traumática nas TC-CE realizadas, reduzindo a exposição desnecessária a radiação ionizante. A atitude expectante preconizada de vigilância clínica em meio hospitalar das crianças e adolescentes com TCE ligeiro, sintomáticos mas sem alterações significativas na observação, não pareceu ter implicado riscos acrescidos. A radiografia de crânio não parece ter utilidade nestes doentes.

\section{AGRADECIMENTOS}

Os autores gostariam de agradecer a António Gomes a sua disponibilidade na revisão crítica do manuscrito e a Paula Azeredo pelo seu entusiasmo na elaboração da norma de orientação clínica nos TCE ligeiros em vigor na nossa unidade hospitalar até ao presente estudo.

\section{PROTECÇÃO DE PESSOAS E ANIMAIS}

Os autores declaram que os procedimentos seguidos estavam de acordo com os regulamentos estabelecidos pelos responsáveis da Comissão de Investigação Clínica e Ética e de acordo com a Declaração de Helsínquia da Associação Médica Mundial.

\section{CONFIDENCIALIDADE DOS DADOS}

Os autores declaram ter seguido os protocolos do seu centro de trabalho acerca da publicação de dados.

\section{CONFLITOS DE INTERESSE}

Os autores declaram não ter quaisquer conflitos de interesse relativamente ao presente artigo.

\section{FONTES DE FINANCIAMENTO}

Não existiram quaisquer fontes externas de financiamento na elaboração do presente trabalho.

head injury in children, young people and adults. NICE clinical guideline January 2014. [accessed 2016 Mar 25]. Available from: https://www. nice.org.uk/guidance/cg176.

8. Pickering A, Harnan S, Fitzgerald P, Pandor A, Goodacre S. Clinical decision rules for children with minor head injury: a systematic review. Arch Dis Child. 2011;96:414-21.

9. Muñoz-Santanach D, Sainz de la Maza VT, Forster GE, Cubells LC Ninos con traumatismo craneal leve en urgencias: inverted question markes necesaria la radiografia de craneo en pacientes menores de 2 anos? Neurocirurgia. 2014;25:149-53.

10. Kacperski J, Arthur T. Management of post-traumatic headaches in children and adolescentes. Headache. 2016;56:36-48.

11. Kuppermann N, Holmes JF, Dayan PS, Hoyle JD Jr, Atabaki SM, Holubkov R, et al. Identification of children at very low risk of clinicallyimportant brain injuries after head trauma: A prospective cohort study. Lancet. 2009;374:1160-70. 
12. Hennelly KE, Mannix R, Nigrovic LE, Lee LK, Thompson KM, Monuteaux $M C$, et al. Pediatric traumatic brain injury and radiation risks: a clinical decision analysis. J Pediatr. 2013;162:392-97.

13. Fundarò C, Caldarelli M, Monaco S, Cota F, Giorgio V, Filoni S, et al. Brain CT scan for pediatric minor acidental head injury. An Italian experience and review of literature. Childs Nerv Syst. 2012;28:1063-8.

14. Esteves I, Crispim J, Farela Neves J, Cunha F. Abordagem do traumatismo craniano ligeiro na idade pediátrica: Neuro-imagem ou atitude conservadora? Acta Pediatr Port. 2009;40:197-202.

15. Schonfeld D, Fitz BM, Nigrovic LE. Effect of the duration of emergency department observation on computed tomography use in children with minor blunt head trauma. Ann Emerg Med. 2013;62:597-603.

16. Wing R, James C. Pediatric head injury and concussion. Emerg Med Clin N Am. 2013;31:653-75.

17. Bainbridge $\mathrm{J}$, Khirwadkar $\mathrm{H}$, Hourihan MD. Vomiting - is this a good indication for CT head scans in patients with minor head injury? $\mathrm{Br} J$ Radiol. 2012;85:183-6.

18. Klassen TP, Reed MH, Stiell IG, Nijssen-Jordan C, Tenenbein M, Joubert $\mathrm{G}$, et al. Variation in utilization of computed tomography scanning for the investigation of minor head trauma in children: A Canadian experience. Acad Emerg Med. 2000;7:739-44.

19. Osmond MH, Klassen TP, Wells GA, Correll R, Jarvis A, Joubert G, et al. CATCH: A clinical decision rule for the use of computed tomography in children with minor head injury. CMAJ. 2010;182:341-8.

20. Nigrovic LE, Lee LK, Hoyle J, Stanley RM, Gorelick MH, Miskin M, et al. Prevalence of clinically important traumatic brain injuries in children with minor blunt head trauma and isolated severe injury mechanisms. Arch Pediatr Adolesc Med. 2012;166:356-61.

21. Mannix R, Meehan WP, Monuteaux MC, Bachur RG. Computed tomography for minor head injury: Variation and trends in major United States pediatric emergency departments. J Pediatr. 2012;160:136-9.

22. Rice HE, Frush DP, Farmer D, Waldhausen $\mathrm{JH}$. Review of radiation risks from computed tomography: essentials for the pediatric surgeon. J Pediatr Surg. 2007;42:603-7.

23. Mathews JD, Forsythe AV, Brady Z, Butler MW, Goergen SK, Byrnes GB, et al. Cancer risk in 680,000 people exposed to computed tomography scans in childhood or adolescence: Data linkage study of 11 million Australians. BMJ. 2013;21:1-18.

24. Tavor O, Boddu S, Kulkarni AV. Presenting characteristics of children who required neurosurgical intervention for head injury. Childs Nerv Syst. 2016;3.

25. Dunning J, Daly JP, Lomas JP, Lecky F, Batchelor J, Mackway-Jones K et al. Derivation of the children's head injury algorithm for the prediction of important clinical events decision rule for head injury in children. Arch Dis Child. 2006;91:885-91.

26. Lyttle MD, Crowe L, Oakley E, Dunning J, Babl FE. Comparing CATCH, CHALICE and PECARN clinical decision rules for paediatric head injuries. Emerg Med J. 2012;29:785-94.

27. Easter JS, Bakes K, Dhaliwal J, Miller M, Caruso E, Haukoos JS Comparison of PECARN, CATCH, and CHALICE rules for children with minor head injury: A prospective cohort study. Ann Emerg Med. 2014;64:145-52.

28. Maguire JL, Boutis K, Uleryk EM, Laupacis A, Parkin PC. Should a head-injured child receive a head CT scan? A systematic review of clinical prediction rules. Pediatrics. 2009;124:145-54.

29. Samuel N, Jacob R, Elion Y, Mashiach T, Shavit I. Falls in young children with minor head injury: a prospective analysis of injury mechanisms. Brain Inj. 2015;29:946-50.

30. Alharthy N, Al Queflie S, Ayousef K, Yunus F. Clinical manifestations that predict abnormal brain computed tomography (CT) in children with minor head injury. J Emerg Trauma Shock. 2015;8:88-93.

31. Nigrovic LE, Schunk JE, Foerster A, Cooper A, Miskin M, Atabaki SM, et al. The effect of observation on cranial computed tomography utilization for children after blunt head trauma. Pediatrics. 2011;127:1067-73.

32. Eisenberg MA, Meehan WP, Mannix R. Duration and course of postconcussive symptoms. Pediatrics. 2014;133:999-1006.

33. Dayan PS, Holmes JF, Hoyle J Jr, Atabaki S, Tunik MG, Lichenstein $\mathrm{R}$, et al. Headache in traumatic brain injuries from blunt head trauma. Pediatrics. 2015;135:504-12.

34. Chelse AB, Epstein LG. Blunt head trauma and headache. Pediatr Neurol Briefs. 2015;29:30.

35. Burns EC, Grool AM, Klassen TP, Correll R, Jarvis A, Joubert G, et al Scalp hematoma characteristics associated with intracranial injury in pediatric minor head injury. Acad Emerg Med. 2016;7.

36. Thiam DW, Yap SH, Chong SL. Clinical decision rules for paediatric minor head injury: are CT scans a necessary evil? Ann Acad Med Singapore. 2015; 44:335-41. 